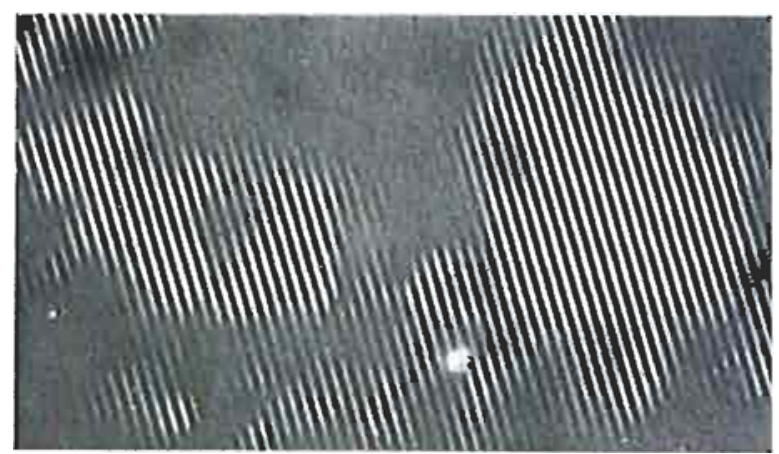

Fig. 4 Condensation of a gold vapour plume is periodically inhibited by the intense standing wave within a laser interferometer and produces lines with spatial period $\mathbf{2 . 5} \mu$

in the gold film has been duplicated in the glass substrate. The amplitude transmittance of such a surface is almost unity but the periodic phase shifts induced by the periodic depth modulation of the glass gives rise to diffraction of incident light.

With a $200 \AA$ gold film the energy required to heat the surface to boiling point and supply the necessary latent heat for vaporisation is about $80 \mathrm{~mJ} / \mathrm{cm}^{2}$ and the high power of a $30 \mathrm{~ns}$ giant pulse was required to accomplish vaporisation before thermal diffusion took place.

Instead of vaporising the gold at the antinodes of the standing wave electric field it has been possible to use the converse effect, i.e. to periodically inhibit the condensation of a gold vapour in the presence of an intense electromagnetic field. To demonstrate this a second plate - the "collector" - was placed parallel to the gold film in Fig. 1. This plate recondensed the gold vapour plume and examination of it revealed a spatially periodic gold layer. Fig. 4 is a micrograph of the collector plate with a spatial period of $2.5 \mu$.

Information recorded on metal surfaces by either of the above techniques has the advantages of increased resolving power, no other processing, "exposure" in full light, and a panchromatic range. Furthermore, because of the short exposure time ( $\sim 30$ ns giant pulse duration) the metal film is more tolerant of vibration and the pattern is permanent.

It is suggested that these techniques could be applied to the manufacture of microcircuits. Deposition of modulated gold and dielectric films might make it possible to fabricate very small capacitors and waveguides. Two crossed gratings might form the basis of a cheap compact electronic memory or storage system.

\section{References}

1 V. I. Little, D. M. Rowley and R. Wiltsher, Nature, 1970, 228, (5266, Oct. 3), 49-50

2 V. I. Little, P. Y. Key, R. Wiltsher and D. M. Rowley, Nature Phys. Sci., 1971, 232, (34, Aug. 23), 165-166

\title{
A New Gold-Lead Compound
}

\section{COMPUTER-AIDED THERMAL ANALYSIS OF SOLDER ALLOYS}

Literally millions of gold-plated electrical and electronic circuit components are joined with the standard soft solders (1). Occasionally problems arise when gold is taken up by the solder and brittle intermetallic compounds are formed, but it has rarely been possible to interpret the structure of the alloys produced in terms of the known equilibrium diagrams of the various binary systems involved. In particular the characteristics of gold-lead-silver alloys were found to be considerably at variance with those suggested by the accepted gold-lead diagram advanced sixty-six years ago by Vogel (2) which showed the presence of two compounds $\mathrm{Au}_{2} \mathrm{~Pb}$ and $\mathrm{AuPb}_{2}$.

The existence of a third compound which forms peritectically at $221^{\circ} \mathrm{C}$ has been recently confirmed by Bhattacharyya and Reynolds (3) of the University of Aston in Birmingham. $\mathrm{AuPb}_{3}$ has a tetragonal crystal structure with lattice constants, $a=11.948 \AA$ and $c=5.878 \AA$. First reported in very thin evaporated gold-lead films by Fujiki $(4,5)$ and later by Jeppesen and Caswell at IBM (6), its existence in massive form has hitherto been doubted.

To facilitate work on the soldering and brazing alloys, investigators at the GEC Hirst Research Centre have recently been applying the principles of automation to thermal analysis. In a paper recently presented at the National Physical Laboratory,
A. Prince et al. (7) reported some of the results of this work. The 14 gold-lead alloys processed by computer yielded in eight days a gold-lead diagram virtually identical with that obtained at Aston by more traditional techniques. The existence of the compound $\mathrm{AuPb}_{3}$ was again confirmed and valuable additional information was reported on the ternary $\mathrm{Au}-\mathrm{Ag}-\mathrm{Sn}$ system.

The savings in labour which result from the application of automation to thermal analysis are therefore very considerable. They augur well for a revival of interest in classical metallography and should certainly lead to a more rational approach in the solution of soldering and brazing problems.

A. S. D.

\section{References}

1 P. A. Ainsworth, Gold Bull., 1971, 4, 47

2 R. Vogel, $Z$. anorg. Chem., 1905, 45, 11

3 J. P. Bhattacharyya and K. A. Reynolds, $\mathcal{f}$. Inst. Metals, $1971,99,350$

4 Y. Fujiki, R. Suganuma and T. Yoshida, 7. Phys. Soc. Japan, 1958, 13, 969

5 Y. Fujiki, Mem. Coll. Sci., Univ. Kyoto (A), 1959, 29, 197

$6 \mathrm{R}$. H. Jeppesen and H. L. Caswell, IBM F., 1963, (October), 297

7 D. S. Evans, J. I. McLeod and A. Prince, Computer Aided Thermal Analysis in the Determination of Phase Relationships, Intl. Symposium on Metallurgical Chemistry, NPL, June, 1971 